\title{
Metabolic profiles predict adverse events after coronary artery bypass grafting
}

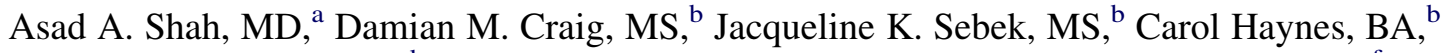 \\ Robert C. Stevens, PhD, ${ }^{c, d}$ Michael J. Muehlbauer, $\mathrm{PhD},{ }^{\mathrm{c}}$ Christopher B. Granger, MD, ${ }^{\mathrm{e}, \mathrm{f}}$ \\ Elizabeth R. Hauser, PhD, ${ }^{\text {, c }}$ L. Kristin Newby, MD, MHS,,${ }^{\text {ef }}$ Christopher B. Newgard, PhD, ${ }^{\text {,d,g }}$ \\ William E. Kraus, MD, ${ }^{\text {ce e }}$ G. Chad Hughes, MD, ${ }^{a}$ and Svati H. Shah, MD, MHS ${ }^{\text {b,c,e }}$
}

Objective: Clinical models incompletely predict the outcomes after coronary artery bypass grafting. Novel molecular technologies can identify biomarkers to improve risk stratification. We examined whether metabolic profiles can predict adverse events in patients undergoing coronary artery bypass grafting.

\begin{abstract}
Methods: The study population comprised 478 subjects from the CATHGEN biorepository of patients referred for cardiac catheterization who underwent coronary artery bypass grafting after enrollment. Targeted mass spectrometry-based profiling of 69 metabolites was performed in frozen, fasting plasma samples collected before surgery. Principal components analysis and Cox proportional hazards regression modeling were used to assess the relation between the metabolite factor levels and a composite outcome of postcoronary artery bypass grafting myocardial infarction, the need for percutaneous coronary intervention, repeat coronary artery bypass grafting, and death.
\end{abstract}

Results: During a mean follow-up period of $4.3 \pm 2.4$ years, 126 subjects $(26.4 \%)$ experienced an adverse event. Three principal components analysis-derived factors were significantly associated with an adverse outcome on univariate analysis: short-chain dicarboxylacylcarnitines (factor 2, $P=.001$ ); ketone-related metabolites (factor $5, P=.02$ ); and short-chain acylcarnitines (factor $6, P=.004$ ). These 3 factors remained independently predictive of an adverse outcome after multivariate adjustment: factor 2 (adjusted hazard ratio, $1.23 ; 95 \%$ confidence interval, $1.10-1.38 ; P<.001$ ), factor 5 (odds ratio, $1.17 ; 95 \%$ confidence interval, $1.01-1.37 ; P=.04$ ), and factor 6 (odds ratio, $1.14 ; 95 \%$ confidence interval, $1.02-1.27 ; P=.03$ ).

Conclusions: Metabolic profiles are independently associated with adverse outcomes after coronary artery bypass grafting. These profiles might represent novel biomarkers of risk that can augment existing tools for risk stratification of coronary artery bypass grafting patients and might elucidate novel biochemical pathways that mediate risk. (J Thorac Cardiovasc Surg 2012;143:873-8)

Coronary heart disease remains the leading cause of death in the United States. Coronary artery bypass grafting (CABG) is the preferred treatment option for the vast majority of patients with left main or 3-vessel coronary artery disease. The beneficial outcomes after CABG, the most widely studied operation in history, are well established. However, the progression of native vessel atherosclerosis and vein graft failure contribute significantly to the continued morbidity and

\footnotetext{
From the Division of Thoracic and Cardiovascular Surgery, ${ }^{\text {a }}$ Department of Surgery; Department of Medicine, ${ }^{b}$ Center for Human Genetics; Sarah W. Stedman Nutrition and Metabolism Center ${ }^{\mathrm{c}}$; Division of Endocrinology, ${ }^{\mathrm{d}}$ Department of Medicine; Division of Cardiovascular Medicine, ${ }^{\mathrm{e}}$ Department of Medicine; Duke Clinical Research Institute ${ }^{f}$; and Department of Pharmacology and Cancer Biology, ${ }^{\mathrm{g}}$ Duke University Medical Center, Durham, NC.

This study was supported by funding from National Institutes of Health grant R01HL095987-01 (to S.H.S.); the Measurement to Understand ReClassification of Disease of Cabarrus and Kannapolis (MURDOCK) Study through a gift to Duke University from the David H. Murdock Foundation; National Institutes of Health grant 1UL1 RR024128-01 from the National Center for Research Resources, a component of the National Institutes of Health and National Institutes of Health Roadmap for Medical Research. Support also received from Division of Cardiovascular and Thoracic Surgery, Duke University Medical Center and the American Heart Association (to S.H.S.).

Disclosures: Authors have nothing to disclose with regard to commercial support.
}

mortality after $\mathrm{CABG}$ in the long term. For example, up to $10 \%$ of patients undergoing CABG require a subsequent medical or surgical revascularization procedure within 3 years after surgery. ${ }^{1}$ Repeat CABG occurs in $11 \%$ of patients by 10 years after the initial CABG. ${ }^{2}$ Furthermore, $14 \%$ to $36 \%$ of patients will have a new postoperative myocardial infarction (MI) within 10 years after their index $\mathrm{CABG},{ }^{3,4}$ with a significant resultant decrease in survival. ${ }^{3}$

The contents are solely the responsibility of the authors and do not necessarily represent the official views of the National Center for Research Resources or National Institutes of Health.

Information on National Center for Research Resources is available from http:// www.ncrr.nih.gov/. Information on Re-engineering the Clinical Research Enterprise is available from http://nihroadmap.nih.gov/clinicalresearch/overview/translational.asp.

Read at the 37th Annual Meeting of The Western Thoracic Surgical Association, Colorado Springs, Colorado, June 22-25, 2011.

Received for publication June 9, 2011; revisions received Aug 18, 2011; accepted for publication Sept 15, 2011; available ahead of print Feb 3, 2011

Address for reprints: Svati H. Shah, MD, Division of Cardiology, Department of Medicine, Duke University Medical Center, DUMC Box 3445, Durham, NC 27710 (E-mail: svati.shah@duke.edu).

$0022-5223 / \$ 36.00$

Copyright $(2012$ by The American Association for Thoracic Surgery doi:10.1016/j.jtcvs.2011.09.070 


$$
\begin{aligned}
& \text { Abbreviations and Acronyms } \\
& \begin{aligned}
\text { CABG } & =\text { coronary artery bypass grafting } \\
\text { CI } & =\text { confidence interval } \\
\text { MI } & =\text { myocardial infarction } \\
\text { OR } & =\text { odds ratio }
\end{aligned}
\end{aligned}
$$

However, clinical models incompletely predict the risk of adverse events after CABG. For example, the European System for Cardiac Operative Risk Evaluation (EuroSCORE) model has a c-statistic of 0.79 and others have a similar model fit. ${ }^{5}$ Furthermore, most currently available risk models predict the perioperative risk but do not describe longer term risk. Thus, a gap remains in our risk prediction models for adverse events after CABG. A better understanding of risk could lead to alternate and/or adjunctive perioperative strategies or the use of alternative initial revascularization strategies to improve the outcomes of patients at high risk of adverse events after CABG. Metabolic profiling, the systematic study of the small molecule byproducts of cellular metabolism, has been used to identify novel cardiovascular biomarkers to improve risk stratification and better understand the mechanisms of several disease processes. ${ }^{6}$ In 1 recent study from our group, a cluster of small-medium chain dicarboxylated acylcarnitine metabolites was predictive of imminent cardiovascular events (death/MI). ${ }^{7}$ We hypothesized that targeted metabolic profiling would identify novel biomarkers of the risk of adverse events after CABG and potentially provide a better understanding of the disease pathophysiology.

\section{METHODS \\ Study Population}

The study population was selected from patients enrolled in the CATHGEN biorepository of more than 9000 subjects at Duke University Medical Center, a biorepository of clinical and follow-up data and blood samples collected from patients presenting for cardiac catheterization. Details of the biorepository have been previously published. ${ }^{8}$ In brief, fasting blood samples were collected in ethylenediamine-tetra-acetic acid tubes using an arterial sheath at cardiac catheterization, before the administration of supplemental heparin (if given). The blood tubes were then chilled to $4{ }^{\circ} \mathrm{C}$, centrifuged within 30 minutes of collection, separated into aliquots, and frozen at $-80^{\circ} \mathrm{C}$. The demographics, medical history, angiographic data, and longitudinal follow-up for the CATHGEN patients were collected through the Duke Databank for Cardiovascular Disease, which has archived this information for all patients undergoing cardiac procedures at the Duke University Medical Center since 1969. The follow-up data include annual determination of mortality, MI, hospitalizations, and coronary revascularization procedures. Vital status is confirmed through the National Death Index and Social Security Death Index.

Metabolic profiling had been performed on 3899 unique subjects from December 2006 to March 2010. ${ }^{7,8}$ For the present study, we selected all subjects with metabolic profiling who underwent CABG at any point after their index CATHGEN cardiac catheterization. The Duke University institutional review board approved the CATHGEN biorepository and the present substudy. Before the collection of blood samples, all study subjects provided written informed consent to participate.

\section{Laboratory Methods}

The levels of 45 acylcarnitines, 15 amino acids, ketones, $\beta$-hydroxybutyrate, and total free fatty acids were quantitatively determined from frozen, fasting plasma samples using previously reported methods with known coefficients of variation. ${ }^{7,9,10}$ The laboratory (Sarah W. Stedman Nutrition and Metabolism metabolomics core laboratory) was kept unaware of the event status, and the samples were randomly numbered with respect to event status. Acylcarnitines and amino acids were profiled using a mass spectrometry-based protocol. ${ }^{9,11}$ The proteins were first removed by precipitation with methanol. Aliquots of supernatants were then dried and esterified with hot, acidic methanol (acylcarnitines) or n-butanol (amino acids). The analysis was done by tandem mass spectroscopy with a Quattro Micro instrument (Waters, Milford, Mass). Quantification of the "targeted" intermediary metabolites was facilitated by the addition of mixtures of known quantities of stable-isotope internal standards, as previously described. ${ }^{7,10,12}$

\section{Statistical Analysis}

The primary endpoint of the present study was occurrence of any event at any point after $\mathrm{CABG}$ from within a composite adverse event, defined as incident percutaneous coronary intervention, $\mathrm{MI}$, repeat $\mathrm{CABG}$, and death.

Principal components analysis was used, given the co-linearity of the metabolites, to reduce the burden of adjustment for multiple comparisons. Metabolites with more than $25 \%$ of the values equaling 0 (less than the lower limits of quantification) were not analyzed (C6 and C7-DC acylcarnitines). Varimax rotation was used to produce identifiable factors. Factors with an eigenvalue of 1 or more were retained according to the commonly used Kaiser criterion. ${ }^{13}$ Metabolites with a factor load of 0.4 or more were reported as constituting a given factor. Scoring coefficients were constructed and used to calculate factor scores for each individual (weighted sum of the standardized metabolites within that factor, weighted on the factor loading for each metabolite).

Cox proportional hazard regression modeling was used to assess the association of factor levels with the interval to the first occurrence of any component of the composite of MI, percutaneous coronary intervention, repeat $\mathrm{CABG}$, or death after the index CABG. Both univariate and multivariate models, adjusted for age, gender, race, dyslipidemia, diabetes, renal disease, smoking, body mass index, baseline ejection fraction, number of diseased coronary vessels at cardiac catheterization, and preprocedural heparin use at sample collection, were performed. The multivariate models also were adjusted for all factors significant on the univariate analyses $(P<.05)$. Clinical variables with $P<.10$ were retained in the final model. For these time-to-event analyses, the day of CABG was considered the baseline time zero. Kaplan-Meier curves, adjusted for all variables retained in the final model, were constructed to visualize the relation of those factors significantly associated with the composite event. The factor levels were divided into tertiles for visualization purposes.

Statistical analysis was performed by 3 of us (D.M.C., A.A.S., and S.H.S.) using SAS version 9.1, (SAS Institute, Cary, NC).

\section{RESULTS}

Of 478 subjects who had metabolic profiling of baseline samples collected at cardiac catheterization and subsequently underwent CABG, 126 (26.4\%) experienced the composite clinical endpoint after CABG during a mean follow-up time of $4.3 \pm 2.4$ years after CABG. Of the 126 patients, $19(4.0 \%)$ experienced MI, $28(5.9 \%)$ underwent percutaneous coronary intervention, $4 \quad(0.8 \%)$ 
TABLE 1. Baseline characteristics of study population

\begin{tabular}{|c|c|c|c|c|}
\hline Characteristic & Overall $(n=478)$ & No event subjects $(n=352)$ & Event subjects $(n=126)$ & $P$ value \\
\hline Age (y) & $61 \pm 11$ & $61 \pm 11$ & $62 \pm 12$ & .33 \\
\hline Gender ( $\%$ female $)$ & $159(33)$ & $111(32)$ & $48(38)$ & .18 \\
\hline Race (\% white) & $355(74)$ & $263(75)$ & $92(73)$ & .71 \\
\hline Diabetes & $170(36)$ & $114(32)$ & $56(44)$ & .02 \\
\hline Dyslipidemia & $309(65)$ & $227(65)$ & $82(65)$ & .91 \\
\hline Renal disease & $12(3)$ & $5(1)$ & $7(6)$ & .02 \\
\hline Current smoker & $262(55)$ & $188(53)$ & $74(58)$ & .30 \\
\hline $\operatorname{BMI}\left(\mathrm{kg} / \mathrm{m}^{2}\right)$ & $30 \pm 7$ & $30 \pm 6$ & $30 \pm 7$ & .69 \\
\hline Ejection fraction $(\%)$ & $54 \pm 14$ & $55 \pm 14$ & $52 \pm 15$ & .03 \\
\hline Diseased coronary vessels (n) & $2.3 \pm 0.8$ & $2.3 \pm 0.8$ & $2.5 \pm 0.8$ & .02 \\
\hline Preprocedure heparin use & $116(24)$ & $77(22)$ & $39(31)$ & .05 \\
\hline
\end{tabular}

Data presented as mean \pm standard deviation or numbers, with percentages in parentheses. BMI, Body mass index.

underwent repeat $\mathrm{CABG}$, and $75(15.7 \%)$ died (first events). The median interval from enrollment in the CATHGEN cardiac catheterization study to CABG was 4 days (interquartile range, 2-49). The baseline characteristics of the study population according to event or no event during follow-up are listed in Table 1.

The principal components analysis identified 12 metabolic factors (Table 2), residing in biologically plausible clusters, as previously reported. ${ }^{7,12,14}$ On univariate timeto-event analyses, the levels of several factors were significantly associated with the interval to the composite event (Table 3). These included factor 2, consisting of shortchain dicarboxylacylcarnitines (hazard ratio [HR], 1.19; $95 \%$ confidence interval $[\mathrm{CI}], 1.07-1.33 ; P=.001)$; factor 5 , consisting of ketone-related metabolites (HR, 1.21; $95 \%$ CI, $1.04-1.41 ; P=.02$ ); and factor 6 , consisting of short- chain acylcarnitines (HR, 1.20; 95\% CI, 1.06-1.35; $P=.004)$.

After multivariate adjustment for the clinical variables and significant factors on univariate analyses, these 3 factors remained significantly associated with the risk of adverse events: factor 2 (HR, 1.23; 95\% CI, 1.10-1.38; $P<.001$ ); factor 5 (HR, 1.17 ; 95\% CI, 1.01-1.37; $P=.04)$; factor 6 (HR, $1.14 ; 95 \%$ CI, 1.02-1.27; $P=.03$; Table 4$)$. The clinical variables, ejection fraction $(P=.05)$ and preprocedure heparin use $(P=.06)$, were also retained in the final model. Kaplan-Meier curves demonstrating the relationship between factor 2 levels and adverse outcomes after CABG are presented in Figure 1, showing a linear relation between metabolite factor levels and outcomes, with greater metabolite factor levels associated with a greater probability of adverse events.

TABLE 2. Principal components analysis

\begin{tabular}{|c|c|c|c|c|}
\hline Factor & Term & Individual components & Eigenvalue & Variance \\
\hline 1 & Medium-chain acylcarnitines & $\begin{array}{l}\mathrm{C} 8, \mathrm{C} 10, \mathrm{C} 10: 1, \mathrm{C} 10-\mathrm{OH} / \mathrm{C} 8-\mathrm{DC}, \mathrm{C} 12, \mathrm{C} 12: 1, \mathrm{C} 14, \mathrm{C} 14: 1, \\
\text { C14:1-OH/C12:1-DC, C14:2, C16:1, C16:1-OH/C14:1-DC, } \\
\text { C16:2, C18:1-OH/C16:1-DC }\end{array}$ & 15.69 & 0.26 \\
\hline 2 & Short-chain dicarboxylacylcarnitines & $\begin{array}{l}\text { Ci4-DC/C4-DC, C5-DC, C6-DC, C6:1-DC/C8:1-OH, C8:1, } \\
\text { C8:1-DC, C10:1, C10:2, C10:3, C10-OH/C8-DC, } \\
\text { C12-OH/C10-DC, citrulline }\end{array}$ & 5.72 & 0.09 \\
\hline 3 & Long-chain dicarboxylacylcarnitines & $\begin{array}{l}\mathrm{C} 12-\mathrm{OH} / \mathrm{C} 10-\mathrm{DC}, \mathrm{C} 14-\mathrm{OH} / \mathrm{C} 12-\mathrm{DC}, \mathrm{C} 16-\mathrm{OH} / \mathrm{C} 14-\mathrm{DC} \\
\mathrm{C} 18-\mathrm{OH} / \mathrm{C} 16-\mathrm{DC}, \mathrm{C} 18: 1-\mathrm{OH} / \mathrm{C} 16: 1-\mathrm{DC}, \\
\text { C20:1-OH/C18:1-DC, C20-OH/C18-DC, C20 }\end{array}$ & 4.41 & 0.07 \\
\hline 4 & Long-chain acylcarnitines & C16, C16:1-OH/C14:1-DC, C18,C18:1, C18:2, C20:4 & 3.17 & 0.05 \\
\hline 5 & Ketone-related & Ketones, Hbut, C4-OH, C2, alanine $(-)$ & 2.83 & 0.05 \\
\hline 6 & Short-chain acylcarnitines & $\mathrm{C} 2, \mathrm{C} 3, \mathrm{C} 4 / \mathrm{Ci} 4 \mathrm{C} 5 \mathrm{~s}$ & 2.52 & 0.04 \\
\hline 7 & Branched-chain amino acids & Leucine/isoleucine, valine, phenylalanine, ornithine & 2.25 & 0.04 \\
\hline 8 & Urea cycle amino acids & Methionine, serine, histidine, citrulline & 1.90 & 0.03 \\
\hline 9 & Amino acids & Glycine, histidine, phenylalanine, C5:1 & 1.74 & 0.03 \\
\hline 10 & Various & Aspartic acid/asparagine, C5:1(-), C5-OH/C3-DC & 1.49 & 0.02 \\
\hline 11 & Medium-chain acylcarnitines & $\mathrm{C} 8: 1, \mathrm{C} 10: 3$ & 1.27 & 0.02 \\
\hline 12 & Various & C22, NEFA & 1.11 & 0.02 \\
\hline
\end{tabular}

Hbut, $\beta$-Hydroxybutyrate; (-), negative factor load for this metabolite on this factor; NEFA, nonesterified fatty acids. 
TABLE 3. Relationship between metabolite factor levels and adverse events

\begin{tabular}{llcccc}
\hline & \multicolumn{1}{c}{ Term } & \multicolumn{3}{c}{ Factor levels } & \\
\cline { 3 - 5 } Factor & & No event subjects $(\mathbf{n}=\mathbf{3 5 2})$ & Event subjects $(\mathbf{n}=\mathbf{~ 1 2 6})$ & HR (95\% CI) & $\boldsymbol{P}$ value* \\
\hline 1 & Medium-chain acylcarnitines & $-0.02 \pm 1.1$ & $0.06 \pm 0.74$ & $1.07(0.94-1.23)$ & .30 \\
2 & Short-chain dicarboxyl-acylcarnitines $\dagger$ & $-0.09 \pm 0.85$ & $0.24 \pm 1.3$ & $1.19(1.07-1.33)$ & .001 \\
3 & Long-chain dicarboxyl-acylcarnitines & $-0.04 \pm 0.81$ & $0.12 \pm 1.39$ & $1.11(0.99-1.24)$ & .08 \\
4 & Long-chain acylcarnitines & $0.03 \pm 1.00$ & $-0.09 \pm 0.99$ & $0.90(0.75-1.08)$ & .25 \\
5 & Ketone-related $\dagger$ & $-0.07 \pm 0.92$ & $0.19 \pm 1.17$ & $1.21(1.04-1.41)$ & .02 \\
6 & Short-chain acylcarnitines $\dagger$ & $-0.07 \pm 0.71$ & $0.19 \pm 1.52$ & $1.20(1.06-1.35)$ & .004 \\
7 & Branched-chain amino acids & $-0.01 \pm 0.98$ & $0.03 \pm 1.06$ & $1.02(0.85-1.21)$ & .87 \\
8 & Urea cycle amino acids & $0.03 \pm 0.97$ & $-0.09 \pm 1.08$ & $0.91(0.72-1.13)$ \\
9 & Amino acids & $-0.001 \pm 1.01$ & $0.002 \pm 0.98$ & $1.15(0.96-1.38)$ & .13 \\
10 & Various & $-0.04 \pm 0.99$ & $0.10 \pm 1.03$ & $1.02(0.86-1.20)$ & .86 \\
11 & Medium-chain acylcarnitines & $-0.05 \pm 0.95$ & $0.14 \pm 1.12$ & $1.16(0.99-1.37)$ & .07 \\
12 & Various & $-0.02 \pm 0.98$ & $0.05 \pm 1.05$ & $1.07(0.90-1.27)$ & .48 \\
\hline
\end{tabular}

$H R$, Hazard ratio; $C I$, confidence interval. $* P$ value for univariate Cox proportional hazards regression model of association of factor level with time-to-event. $\dagger$ Metabolite factors significantly associated with time-to-event.

Kaplan-Meier curves demonstrating the relation between factors 5 and 6 with adverse event are presented in Figures 2 and 3.

\section{DISCUSSION}

To our knowledge, this is the first report of an association between the baseline plasma metabolic profiles and outcomes after CABG. In this cohort, elevated levels of short-chain dicarboxylacylcarnitines, ketone-related metabolites, and short-chain acylcarnitines were predictive of a composite endpoint of MI, repeat revascularization, or death at any point after CABG. This predictive capacity held true after adjustment for numerous clinical covariates known to be associated with cardiovascular events.

Our group has previously observed that elevated levels of short-chain dicarboxylacylcarnitines are independently associated with a greater risk of death and incident MI for those undergoing cardiac catheterization. ${ }^{7,14}$ Additionally, we have previously shown that adding the metabolic profiles to the clinical risk assessment tools can significantly improve risk stratification and help reclassify patients with intermediate-risk coronary artery disease. ${ }^{7}$ The results of the present study have extended those findings into this specific subgroup of patients with coronary artery disease who experience adverse events after

TABLE 4. Multivariate model for time to adverse event

\begin{tabular}{lcc}
\hline \multicolumn{1}{c}{ Variable } & HR $(\mathbf{9 5} \% \mathbf{C I})$ & Adjusted $\boldsymbol{P}$ value* \\
\hline Factor 2 & $1.23(1.10-1.38)$ & $<.001$ \\
Factor 5 & $1.17(1.01-1.37)$ & .04 \\
Factor 6 & $1.14(1.02-1.27)$ & .03 \\
Ejection fraction & $0.99(0.98-1.00)$ & .05 \\
Preprocedure heparin use & $1.43(0.98-2.10)$ & .06 \\
\hline
\end{tabular}

$H R$, Hazard ratio; $C I$, confidence interval. *Adjusted for age, sex, race, hyperlipidemia, diabetes, renal disease, smoking, body mass index, ejection fraction, number of diseased coronary vessels, preprocedural heparin use, and factors significant on univariate analyses (factors 2,5 , and 6).
CABG, patients with a more severe disease burden in a group with a more homogeneous degree of coronary artery disease severity.

Dicarboxylated acylcarnitines can be derived by $\omega$-oxidation of fatty acids by way of microsomal cytochrome p450 enzymes. Long-chain dicarboxylated fatty acyl coenzyme A ( $\mathrm{CoA})$ is shortened mainly by peroxisomal oxidation ${ }^{15}$ and, when converted to the cognate acylcarnitines, is diagnostic for several inborn errors of metabolism. ${ }^{16}$ Hence, factor 2 might reflect changes in flux through the microsomal cytochrome $\mathrm{p} 450$ and/or peroxisomal oxidation systems. However, dicarboxylated acylcarnitines can also be derived from CoA esters generated during oxidation of amino acids or other metabolic fuels in the mitochondria. Thus, the pathways by which these metabolites are generated and their biologic significance remain to be investigated.

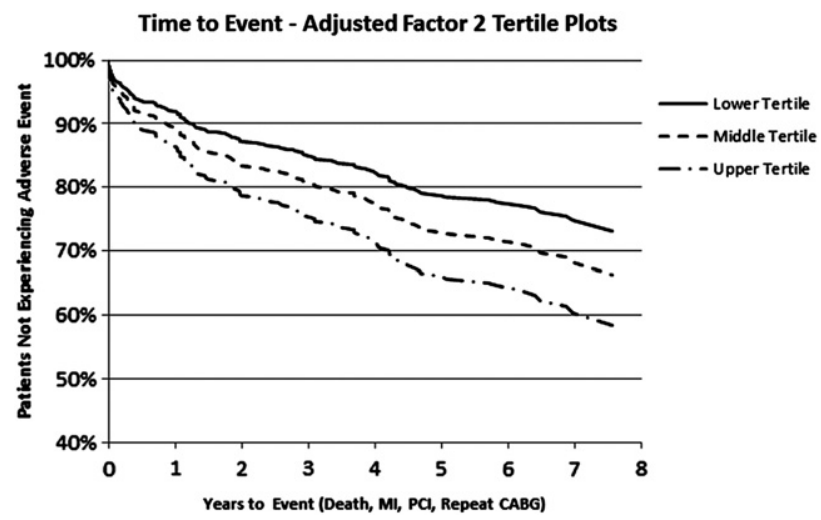

FIGURE 1. Kaplan-Meier curve for relationship between factor 2 levels and adverse events showing adjusted Kaplan-Meier curve of relationship between tertiles of metabolite factor 2 and event-free survival after coronary artery bypass grafting $(C A B G)$, adjusted for all variables retained in final multivariate model, demonstrating linear relationship between factor 2 levels and decreased event-free survival. MI, Myocardial infarction; PCI, percutaneous coronary intervention. 


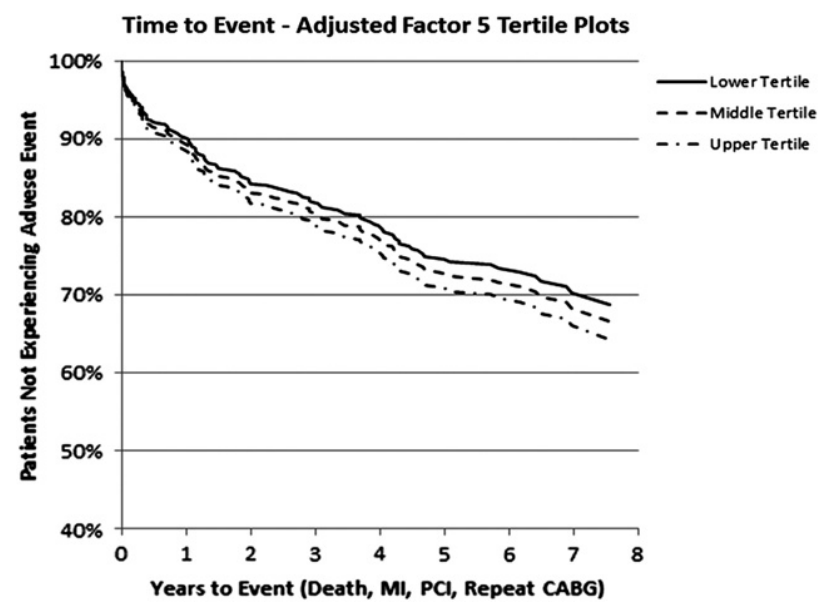

FIGURE 2. Kaplan-Meier curve for relationship between factor 5 levels and adverse events showing adjusted Kaplan-Meier curve of relationship between tertiles of metabolite factor 5 and event-free survival after coronary artery bypass grafting $(C A B G)$, adjusted for all variables retained in final multivariate model, demonstrating linear relationship between factor 5 levels and decreased event-free survival. MI, Myocardial infarction; PCI, percutaneous coronary intervention.

Short chain acylcarnitines (factor 6) are also products of fatty acid oxidation. Similar to dicarboxylacylcarnitines, elevated systemic levels of short chain acylcarnitines can be indicative of inborn errors of metabolism. For example, butyrylcarnitine is elevated in patients with short-chain acyl-CoA dehydrogenase deficiency. The elevated levels of factor 6 in our adverse event population could also have be related to errors in fatty acid oxidation.

In addition to acylcarnitines, a group of ketone-related metabolites were predictive of adverse outcomes in our study. During normal conditions, the heart preferentially oxidizes fatty acids for energy. However, during periods of metabolic stress, such as during an ischemic event, glucose becomes the predominant source of fuel, which is protective. ${ }^{17,18}$ Failure of a cell to increase glucose uptake can result in cellular dysfunction. ${ }^{19}$ Increased levels of ketones (1) correlate with the level of cardiac dysfunction ${ }^{20}$; (2) increase reactive oxygen species production ${ }^{17}$; and (3) decrease glucose uptake. ${ }^{17}$ These mechanisms could explain the increased levels of ketone-related metabolites in patients experiencing adverse events after CABG.

There are potential implications of these findings. First, with respect to clinical applicability, our results suggest that the metabolic profiling of patients undergoing CABG could provide healthcare providers an additional tool for identifying patients at greater risk of adverse events, facilitating personalized tailoring of the revascularization and postprocedure treatment of these patients. For example, patients with greater preoperative plasma levels of shortchain dicarboxylacylcarnitines could benefit from total arterial revascularization, postoperative clopidogrel use

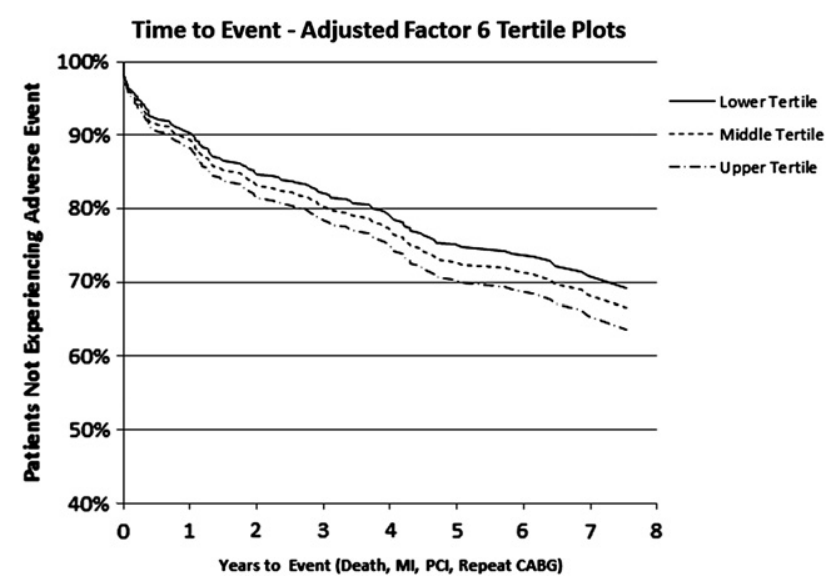

FIGURE 3. Kaplan-Meier curve for relationship between factor 6 levels and adverse events showing adjusted Kaplan-Meier curve of relationship between tertiles of metabolite factor 6 and event-free survival after coronary artery bypass grafting $(C A B G)$, adjusted for all variables retained in final multivariate model, demonstrating linear relationship between factor 6 levels and decreased event-free survival. MI, Myocardial infarction; PCI, percutaneous coronary intervention.

(instead of aspirin), and closer postdischarge follow-up. Although we are optimistic about such a role of these novel biomarkers, such applications necessitate additional studies, including further validation and prospective outcome studies. Second, specific metabolites and metabolic pathways might provide insight into novel mechanisms of disease in patients who experience adverse events after CABG. We have previously observed that metabolic profiles are heritable, ${ }^{12}$ and genetic variation has been shown to be associated with metabolic traits ${ }^{21}$; thus, the identified metabolic profiles might actually be reporting on underlying genetic variations that increase the risk of adverse events after CABG. Both of these implications provide fertile opportunity for additional study.

The limitations of the present study are worth noting. First, although blood was drawn from all study subjects before $\mathrm{CABG}$, potential variability existed in the clinical states of these patients at sampling. For example, patients experiencing non-ST-segment elevation myocardial infarction might have different metabolite profiles than patients undergoing elective cardiac catheterization. However, we did adjust for preprocedural heparin use as a surrogate, because most patients with non-ST-segment elevation myocardial infarction would be treated with heparin before cardiac catheterization. Second, although mass spectrometry is a readily available technology, the quantification of the plasma concentrations of these metabolites involved the use of a specialized center experienced in performing these assays. Many clinical laboratories might not have the capabilities to perform these assays in a timely fashion at present. Work is ongoing to translate these findings into more readily available clinical tools, including 
determining the optimal time to obtain these metabolite profiles preoperatively. Other limitations included the bias of not having enrolled unstable patients who were unable to undergo the consent process because of their instability (ie, ST-segment elevation MI), and the nonrandomized selection of patients.

In conclusion, we have identified metabolic profiles composed of short-chain dicarboxylacylcarnitines, ketonerelated metabolites, and short-chain acylcarnitines that predict adverse outcomes after CABG independent of standard clinical predictors of risk. These findings suggest that metabolites could be significant cardiovascular risk biomarkers that might shed light on novel biochemical pathways of disease and supplement clinical risk assessment models in patients undergoing CABG.

We would like to thank the patients who participated in CATHGEN and Elaine Dowdy for her study coordination; and Melissa Hurdle, Amanda House, and Becky Church for sample collection.

\section{References}

1. Kimura T, Morimoto T, Furukawa Y, Nakagawa Y, Shizuta S, Ehara N, et al. Long-term outcomes of coronary-artery bypass graft surgery versus percutaneous coronary intervention for multivessel coronary artery disease in the bare-metal stent era. Circulation. 2008;118(14 Suppl):S199-209.

2. Cosgrove DM, Loop FD, Lytle BW, Gill CC, Golding LA, Gibson C, et al. Predictors of reoperation after myocardial revascularization. J Thorac Cardiovasc Surg. 1986;92:811-21.

3. Sergeant PT, Blackstone EH, Meyns BP. Does arterial revascularization decrease the risk of infarction after coronary artery bypass grafting? Ann Thorac Surg. 1998;66:1-11

4. Peduzzi P, Detre K, Murphy ML, Thomsen J, Hultgren H, Takaro T. Ten-year incidence of myocardial infarction and prognosis after infarction. Department of Veterans Affairs Cooperative Study of Coronary Artery Bypass Surgery. Circulation. 1991;83:747-55.

5. Nashef SA, Roques F, Michel P, Gauducheau E, Lemeshow S, Salamon R. European System for Cardiac Operative Risk Evaluation (EuroSCORE). Eur J Cardiothorac Surg. 1999;16:9-13.
6. Suhre K, Meisinger C, Döring A, Altmaier E, Belcredi P, Gieger C, et al. Metabolic footprint of diabetes: a multiplatform metabolomics study in an epidemiological setting. PLoS One. 2010;5:e1395.

7. Shah SH, Bain JR, Muehlbauer MJ, Stevens RD, Crosslin DR, Haynes C, et al. Association of a peripheral blood metabolic profile with coronary artery disease and risk of subsequent cardiovascular events. Circ Cardiovasc Genet. 2010;3: 207-14.

8. Shah SH, Granger CB, Hauser ER, Kraus WE, Sun JL, Pieper K, et al. Reclassification of cardiovascular risk using integrated clinical and molecular biosignatures: design of and rationale for the Measurement to Understand the Reclassification of Disease of Cabarrus and Kannapolis (MURDOCK) Horizon 1 Cardiovascular Disease Study. Am Heart J. 2010;160:371-9.e2.

9. An J, Muoio DM, Shiota M, Fujimoto Y, Cline GW, Shulman GI, et al. Hepatic expression of malonyl-CoA decarboxylase reverses muscle, liver and wholeanimal insulin resistance. Nat Med. 2004;10:268-74.

10. Newgard CB, An J, Bain JR, Muehlbauer MJ, Stevens RD, Lien LF, et al. A branched-chain amino acid-related metabolic signature that differentiates obese and lean humans and contributes to insulin resistance. Cell Metab. 2009;9: 311-26. erratum in Cell Metab. 2009;9:565-6.

11. Chace DH, Hillman SL, Millington DS, Kahler SG, Roe CR, Naylor EW. Rapid diagnosis of maple syrup urine disease in blood spots from newborns by tandem mass spectrometry. Clin Chem. 1995;41:62-8.

12. Shah SH, Hauser ER, Bain JR, Muehlbauer MJ, Haynes C, Stevens RD, et al. High heritability of metabolomic profiles in families burdened with premature cardiovascular disease. Mol Syst Biol. 2009;5:258.

13. Kaiser HF. The application of electronic computers to factor analysis. Educ Psychol Meas. 1960;20:141-51.

14. Shah SH, Sun JL, Pieper K, Crosslin DR, Haynes C, Bain JR, et al. Plasma metabolic profiles predict future cardiovascular events. Circulation. 2009;120:S466-7.

15. Ferdinandusse S, Denis S, Van Roermund CW, Wanders RJ, Dacremont G. Identification of the peroxisomal beta-oxidation enzymes involved in the degradation of long-chain dicarboxylic acids. J Lipid Res. 2004;45:1104-11.

16. Johnson DW. Synthesis of dicarboxylic acylcarnitines. Chem Phys Lipids. 2004; 129:161-71.

17. Pelletier A, Coderre L. Ketone bodies alter dinitrophenol-induced glucose uptake through AMPK inhibition and oxidative stress generation in adult cardiomyocytes. Am J Physiol Endocrinol Metab. 2007;292:E1325-32.

18. Depre C, Vanoverschelde JLJ, Taegtmeyer H. Glucose for the heart. Circulation. 1999;99:578-88.

19. Abdel-Aleem S, St Louis JD, Hughes GC, Lowe JE. Metabolic changes in the normal and hypoxic neonatal myocardium. Ann N Y Acad Sci. 1999;874:254-61.

20. Lommi J, Kupari M, Koskinen P, Naveri H, Leinonen H, Pulkki K, et al. Blood ketone bodies in congestive heart failure. J Am Coll Cardiol. 1996;28:665-72.

21. Illig T, Gieger C, Zhai G, Römisch-Margl W, Wang-Sattler R, Prehn C, et al. A genome-wide perspective of genetic variation in human metabolism. Nat Genet. 2010;42:137-41. 